\title{
ESTIMATIVA DO COEFICIENTE DE CULTURA (Kc) DO FEIJOEIRO EM ILHA SOLTEIRA, SP
}

\author{
João Alberto Fischer Filho ${ }^{1}$, João Luís Zocoler ${ }^{2}$
}

\begin{abstract}
RESUMO
O feijoeiro, cultivado em praticamente todas regiões brasileiras, tem a irrigação como uma técnica imprescindível quando a semeadura é realizada no inverno, assim o coeficiente de cultura (Kc) para ser utilizado no manejo da irrigação pode ser obtido por meio de um modelo ajustado através da distribuição em função do tempo dos seus valores médios. A proposta deste trabalho é determinar os valores de Kc em ambiente protegido e comparar dois intervalos de reposição de água na região de Ilha Solteira, SP. O experimento foi conduzido em vasos, os tratamentos constaram de dois diferentes intervalos de reposição de água com quatro repetições. A evapotranspiração da cultura (ETc) foi determinada pela diferença entre as pesagens diárias dos vasos, a evapotranspiração de referência (ETo) foi estimada pelo método de Penman-Monteith, e o Kc foi determinado pela relação entre a ETc e ETo. Os valores de Kc apresentaram-se distintos nos diferentes estágios da cultura, diferiram entre os tratamentos e apresentaram curva característica do Kc. Conclui-se que o tratamento sem reposição diária de água apresentou maior precisão, acurácia e tendência quando comparado ao tratamento com adição diária de água, e os valores de Kc diferiram daqueles propostos por Doorenbos e Kassam ressaltando a necessidade da determinação de valores regionais de Kc.
\end{abstract}

Palavras-chave: curva característica, evapotranspiração, irrigação, Phaseolus vulgaris

ABSTRACT

ESTIMATED CROP COEFFICIENT (Kc) BEAN IN ILHA SOLTEIRA, SP

Bean plants grown in almost all regions of Brazil, have irrigation as an essential technique when seeding is performed in the winter, so the crop coefficient $(\mathrm{Kc})$ to be used for irrigation management can be obtained by means of an adjusted model through distribution versus time their average values. The purpose of this study is to determine Kc values in a protected environment and compare two water replacement intervals in Ilha Solteira region, SP. The experiment conducted in pots; treatments consisted of two different water replacement intervals with four replications. The crop evapotranspiration (ETc) was determined by the difference between the daily weighing of the pots, the reference evapotranspiration (ETo) was estimated by the Penman-Monteith method, and the Kc was determined by the ratio between ETc and ETo. Kc values were presented different at different stages of culture, they differed between treatments and presented characteristic curve of Kc. We conclude that treatment without daily replacement of water showed higher precision, accuracy and tendency when compared to treatment with daily addition of water, and the Kc values differ from those proposed by Doorenbos and Kassam emphasizing the need for determination of regional Kc values.

Keywords: characteristic curve, evapotranspiration, irrigation, Phaseolus vulgaris

\section{Recebido para publicação em 25/02/2016. Aprovado em 24/05/2016.}

1 - Engenheiro Agrônomo, Doutorando em Agronomia (Ciência do Solo), FCAV - UNESP/Jaboticabal. joaofischer16@gmail.com.

2 - Engenheiro Agrônomo, Professor Adjunto, FEIS - UNESP/Ilha Solteira. 


\section{INTRODUÇÃO}

O feijoeiro apresenta grande importância socioeconômica por ser cultivado por produtores de pequeno a grande porte em praticamente todo território brasileiro. Entretanto, os diferentes níveis de tecnologia usados, a instabilidade da produção proporcionada pelas diversas condições climáticas, como a deficiência hídrica (GUIMARÃES et al., 2006), e a possibilidade de cultivo o ano inteiro contribuem para a baixa produtividade média, estimada em $1.132 \mathrm{~kg} \mathrm{ha}^{-1}$ (CONAB, 2015). Sendo assim, o manejo racional da irrigação, com a utilização do coeficiente de cultura, reduz o efeito da má distribuição de chuvas e possibilita maiores rendimentos e estabilidade da produção.

A evapotranspiração de qualquer cultura é uma das principais informações necessárias para o manejo racional da irrigação e para fins de planejamento do uso da água. Dentre as abordagens disponíveis para a estimativa do consumo de água pelas plantas, destaca-se o uso do coeficiente de cultura (Kc) (MENDONÇA et al., 2007), o qual se torna ferramenta indispensável para utilização pelos irrigantes em culturas agrícolas. A distribuição temporal do Kc constitui uma curva da cultura, obtida experimentalmente, e integra o efeito da mudança na área da folha, da altura da planta, do grau de cobertura e da resistência do dossel da planta sobre a evapotranspiração da cultura (ETc) (OLIVEIRA, 2003).

O conhecimento do coeficiente de cultura é fundamental para se manejar a irrigação e, uma vez que esses valores variam conforme a disponibilidade energética do local, variedade e idade da planta é importante a obtenção de valores regionalizados (SILVA et al., 2006). Ao determinar o coeficiente de cultura para o feijão-fava, Simeão et al. (2013) verificaram que o Kc apresentou valor mínimo de 0,87 no início do desenvolvimento vegetativo, máximo de 1,55 durante a frutificação e 1,38 no final do ciclo da cultura. Ao estudar o consumo de água do feijoeiro irrigado em sistema de plantio direto e convencional, Bizari et al. (2009) verificaram menores lâminas de irrigação para as plantas em plantio direto e estas apresentaram menores coeficientes de cultura. Em vista disso, é fundamental a adoção de mecanismos que favoreçam o aumento da eficiência do uso da água, sem que a produtividade das culturas seja afetada (SOUZA et al., 2011).

Valores de Kc têm sido recomendados pela Food and Agriculture Organization (FAO), entretanto estes compreendem dados médios obtidos para diferentes regiões do mundo que levam em consideração a duração dos estádios fenológicos da cultura que pode variar em função do clima local (RIBEIRO et al., 2009; BASTOS et al., 2008). Portanto, são necessários trabalhos regionais que estudem aspectos como a quantidade de água a ser aplicada de acordo com cada cultivar e solo, os $\mathrm{Kc}$ a serem usados nas diversas fases e a resposta da cultura a irrigação, possibilitando assim o consumo consciente e sustentável de água.

Partindo da hipótese que diferentes épocas de irrigação afetam o coeficiente de cultura do feijoeiro, o presente trabalho foi planejado com o objetivo de estimar os valores de Kc em ambiente protegido comparando dois intervalos de reposição de água na região de Ilha Solteira, SP; e comparar os valores de Kc obtidos com aqueles disponíveis na literatura.

\section{MATERIAL E MÉTODOS}

O trabalho foi realizado em casa de vegetação, no Campus II da Faculdade de Engenharia de Ilha Solteira, da Universidade Estadual Paulista (UNESP). Foi utilizado o feijão carioca cultivar BRSMG Majestoso, com semeadura feita em 24 de junho de 2012.

O solo para o ensaio, antes de serem extraídas as amostras, foi peneirado (peneira com malha de $4 \mathrm{~mm}$ ), homogeneizado e secado até atingir a umidade residual. Depois, o solo nos vasos foi saturado e deixado drenar até a umidade da capacidade de campo, sendo coberta a parte superior dos mesmos para evitar a perda por evaporação durante o processo de drenagem. Ao se obter pesagens sucessivas constantes, calculouse a umidade à capacidade de campo. Amostras do solo foram enviadas aos laboratórios para análise de fertilidade, análise granulométrica e para a obtenção da umidade do solo no ponto de murcha permanente.

Após o plantio, os vasos foram mantidos em condições adequadas de desenvolvimento até a fase fenológica V3 (primeira folha trifoliada), deixando em seguida uma planta por vaso. As pesagens dos

\section{REVENG}


vasos foram feitas diariamente, e sempre que se fez necessária a reposição de água, o volume aplicado foi com referência à diferença de pesagens. Desta maneira, foi possível a determinação da ETc diária, em função da diferença de pesagens.

Ressalta-se que os tratamentos se diferiram de acordo com a reposição de água:

- Tratamento $1(\mathrm{Kc} 1)$ : foi realizada a reposição diária de água de $100 \%$ ETc.

- Tratamento 2 (Kc2): reposição de 100\% da evapotranspiração, medida num período cuja depleção da água no solo foi $60 \%$ da água disponível (AD), não ocorrendo diariamente.

Foram realizadas uma adubação de plantio e duas adubações nitrogenadas em cobertura no momento em que as plantas apresentaram três folhas trifoliadas e no início do enchimento de grãos, seguindo recomendações de Raij et al. (1997). E controle químico de pragas e doenças e manual de plantas de daninhas.

Os valores da evapotranspiração de referência (ETo) foram obtidos por meio dos dados climáticos da Estação Agrometeorológica de Ilha Solteira (UNESP, 2012) localizada próxima ao experimento, e os dados foram estimados pelo método de Penman-Monteith que é considerado padrão pela Food and Agriculture Organization - FAO (ALLEN et al., 1998). Os valores de Kc foram calculados pela razão entre a ETc e a ETo.

$\mathrm{O}$ ciclo da cultura foi dividido em quatro fases: I: semeadura até $10 \%$ da cobertura do solo; II: $10 \%$ da cobertura do solo até $80 \%$ da cobertura do solo; III: $80 \%$ da cobertura do solo até início do amadurecimento; IV: início do amadurecimento até a colheita. $\mathrm{E}$ os resultados obtidos foram comparados àqueles indicados por Doorenbos e Kassam (1979).

Foram realizadas análises de regressão para determinação da equação que melhor representa os valores de Kc durante o ciclo da cultura. As seguintes análises estatísticas foram realizadas para avaliar a precisão e para comparar os modelos: acurácia utilizou-se a eficiência da modelagem (EF), precisão foi medida pelo coeficiente de determinação $\left(\mathrm{R}^{2}\right)$, e tendência avaliada pelo erro médio da estimativa (EME). A EF e o EME foram calculados com as equações 1 e 2 , respectivamente:

$$
E=1-\frac{\sum(\text { Yobs }- \text { Yest })^{2}}{\sum(\text { Yobs }-\bar{Y})^{2}}
$$

em que,

Yobs = coeficiente de cultura observado; Yest $=$ coeficiente de cultura estimado; $\mathrm{e}$ $\overline{\mathrm{Y}}=$ coeficiente de cultura estimado médio.

$\mathrm{EME}=1-\frac{\sum(\text { Yobs }-\overline{\mathrm{Y}})}{\mathrm{N}}$

em que,

Yobs = coeficiente de cultura observado; $\overline{\mathrm{Y}}=$ coeficiente de cultura estimado médio; e $\mathrm{N}=$ número de observações.

\section{RESULTADOS E DISCUSSÃO}

O ciclo da cultura foi de 94 dias, o qual foi divido em cinco fases com diferente duração (Quadro 1), ressalta-se que a última fase (maturação a colheita) foi a mais extensa com 32 dias, entretanto a fase III (maior desenvolvimento vegetativo e início do amadurecimento) foi a qual apresentou maior evapotranspiração para ambos tratamentos, destacando-se com maior necessidade de água.

Quadro 1. Período somatório da evapotranspiração da cultura (ETc) e da evapotranspiração de referência (ETo), em mm para cada fase de desenvolvimento da cultura do feijoeiro, Ilha Solteira, SP

\begin{tabular}{ccccccc}
\hline Fase/período & & I & II & III & IV & Total \\
\hline Início & & $24 / 06 / 2012$ & $03 / 07 / 2012$ & $01 / 08 / 2012$ & $25 / 08 / 2012$ & \\
& Kc1 & 7,7 & 105,1 & 136,4 & 139,0 & 399,6 \\
ETc (mm) & Kc2 & 7,2 & 99,1 & 135,0 & 125,0 & 377,7 \\
& & 9,2 & 93,7 & 120,7 & 156,6 & 398,6 \\
\hline
\end{tabular}


O tratamento que teve reposição de água diária da evapotranspiração apresentou maior ETc em todas as fases do ciclo da cultura e encerrou o seu ciclo com ETc total de 399,6 mm, destaca-se que o mesmo recebeu uma lâmina média de água de 393 $\mathrm{mm}$. Já o tratamento que teve aplicação em diferentes dias apresentou ETc de 377,7 mm no ciclo total, com lâmina total média de $367 \mathrm{~mm}$. Valores próximos foram observados por Lima et al. (2011) que, ao estudarem a evapotranspiração do feijão-caupi em condições de sequeiro, observaram ETc total de 330,7 $\mathrm{mm}$, com a fase reprodutiva com maior ETc.

Os valores de Kc apresentaram valores distintos nas cincos fases de desenvolvimento e, entre os tratamentos (Quadro 2), destaca-se o com reposição diária de água que apresentou valores superiores em todas as fases.

No primeiro estágio, o Kc apresentou valores 0,70 e 0,67 para os tratamentos Kc1 e Kc2 respectivamente, caracterizando-se como os valores mais baixos entre os estágios de desenvolvimento da planta, sendo influenciado, principalmente, pela demanda evaporativa do solo devido ao início de desenvolvimento da planta e, consequentemente, à pequena fração do solo sombreada pela planta. Resultados semelhantes foram observados por Bastos et al. (2008) que, ao avaliarem o coeficiente de cultivo do feijão-caupi, verificaram no estágio inicial um Kc de 0,80 .

$\mathrm{Na}$ fase II, caracterizada pelo pleno desenvolvimento vegetativo, a planta requer um maior volume de água para realização de suas principais atividades metabólicas, e ocorre o aumento da evapotranspiração, assim o Kc encontrado foi 1,12 e 1,06.

Na fase III, em razão da floração e enchimento de vagens, a planta exige maior disponibilidade hídrica e a ETc chega ao seu ápice, os valores de Kc nesta fase praticamente se igualaram entre os tratamentos, com 1,13 e 1,12. Ao avaliarem a supressão de água em diferentes fases fenológicas do feijoeiro, Miorini et al. (2011) verificaram que os tratamentos mais afetados no tocante à produtividade foram o de supressão da irrigação na fase vegetativa e na floração, sendo que não diferiram da supressão de irrigação em todas as fases.

E, por fim, a fase IV apresentou Kc inferior as fases II e III, pois tem como característica a senescência da planta, ou seja, o final do ciclo da cultura, apresentando menores valores de evapotranspiração diária, assim o Kc obtido foi 0,89 e 0,80 .

Quando comparados os valores obtidos com aqueles indicados por Doorenbos e Kassam (1979), nota-se que, embora apresentem uma tendência, estes são diferentes (Quadro 2). Como estes pesquisadores obtiveram os valores médios de diversos experimentos, em diferentes regiões, além de cultivares diferentes, os valores se diferenciam. Deve-se ressaltar ainda que as condições de trabalho dos autores citados foram diferentes daquelas apresentadas neste trabalho.

Quadro 2. Coeficientes da cultura médios $(\mathrm{Kc})$ para diferentes estágios de desenvolvimento do feijoeiro, indicados por Doorenbos e Kassam (1979), Santana et al. (2008) e atual experimento, Ilha Solteira, SP.

\begin{tabular}{ccccc}
\hline Estágios & Kc 1 & Kc 2 & $\begin{array}{c}\text { Doorenbos e Kassam } \\
(1979)\end{array}$ & Santana et al. (2008) \\
\hline Inicial & - & - & - & 0,50 \\
I & 0,70 & 0,67 & 0,30 & 0,53 \\
II & 1,12 & 1,06 & 0,70 & 0,81 \\
III & 1,13 & 1,12 & 1,05 & 1,07 \\
IV & 0,89 & 0,80 & 0,65 & 0,78 \\
\hline
\end{tabular}


Corroborando com os resultados (Quadro 2), Santana et al. (2008), determinando o coeficiente de cultura do feijoeiro em Pato de Minas, MG, obtiveram valores médios de Kc mais próximos ao atual experimento quando comparado aos valores determinados por Doreenbos e Kassam (1979) evidenciando a importância da obtenção do Kc para as condições locais.

Para a maioria das culturas, o valor de $\mathrm{Kc}$ aumenta desde um valor mínimo, na germinação, até um valor máximo, quando a cultura atinge seu pleno desenvolvimento, decrescendo após o início da maturação (OLIVEIRA, 2003), comportamento observado para o feijão carioca, cultivar BRSMG (Figura 1).

A partir dos valores de Kc, foram aplicadas análises de regressão e realizadas medidas de precisão, eficiência da modelagem (Equação 1) e erro médio da estimativa (Equação 2) para comparação entre os tratamentos, os valores são apresentados na Quadro 3. O tratamento que teve reposição de água em diferentes dias apresentou valores de precisão, acurácia e tendência superiores aquele que teve aplicação diária de água.
Para o cultivo do feijoeiro em inverno na região de Ilha Solteira/SP, o modelo com reposição de água quando o teor de água disponível do solo alcançar, aproximadamente, $60 \%$ da capacidade de campo apresentou melhores resultados com valores superiores nas medidas de precisão realizadas. Apresentando valores próximos a $90 \%$ em eficiência da modelagem e coeficiente de determinação, e valor próximo a zero no erro médio da estimativa. Portanto, a estimativa do coeficiente de cultura do feijoeiro em casa de vegetação apresentou excelente eficiência quando utilizado manejo sustentável, ou seja, sem aplicação diária da água para irrigação.

O modelo com reposição diária de água apresentou valores inferiores ao anterior, entretanto destaca-se que a eficiência de modelagem e coeficiente de determinação foram superiores a $80 \%$ e o erro próximo a zero. Corroborando com os resultados, Leal e Sediyama (2004), ao ajustarem modelos matemáticos para determinação da curva do Kc, observaram valores de coeficiente de determinação superiores a 90\% e concluíram que o polinômio de terceiro graus apresentou, em geral, menores valores de erro relativo.
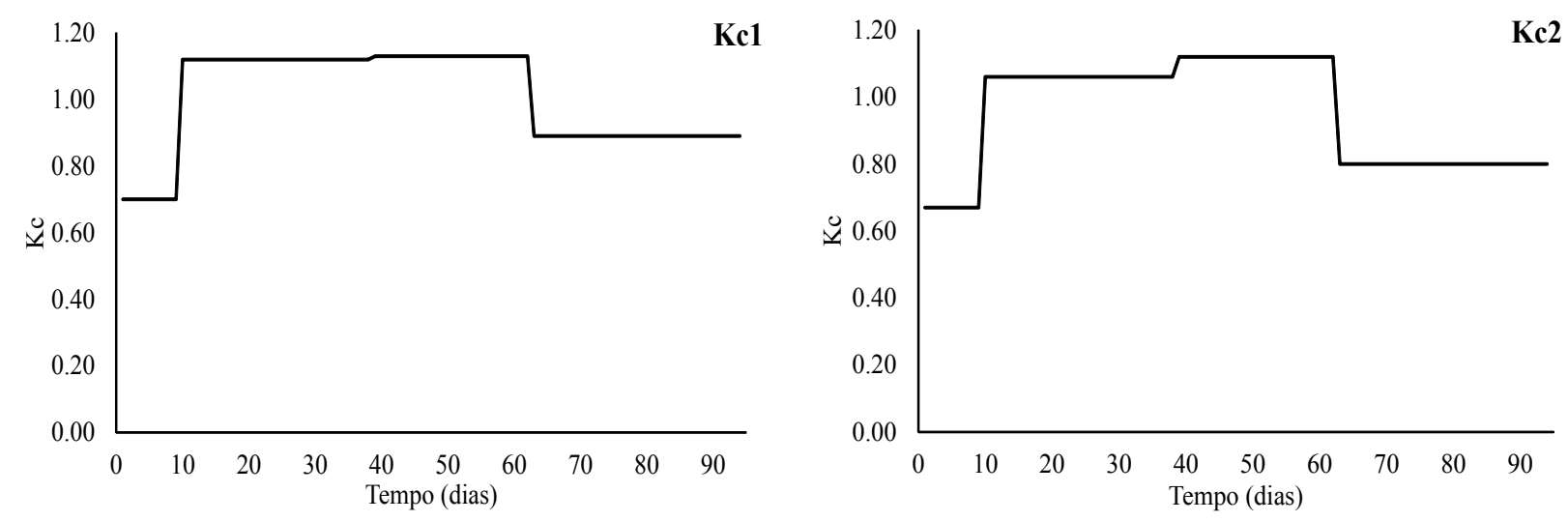

Figura 1. Coeficiente de cultura $(\mathrm{Kc})$ médio observado durante a condução do experimento

Quadro 3. Análises de regressão e medidas de precisão para o coeficiente de cultura, Ilha Solteira, SP

\begin{tabular}{ccccc}
\hline & \multirow{2}{*}{ Equação } & \multicolumn{2}{c}{$\mathrm{EF}$} & \multicolumn{2}{c}{$\mathrm{R}^{2}$} & \multirow{2}{*}{ EME } \\
\cline { 3 - 3 } & $\mathrm{Kc}=-0,0002 \mathrm{x}^{2}+0,0229 \mathrm{x}+0,5166$ & 80,5 & 89,5 & 0,00027 \\
$\mathrm{Kc1}$ & $\mathrm{Kc}=-0,0002 \mathrm{x}^{2}+0,0237 \mathrm{x}+0,4729$ & 90,1 & 92,7 & 0,00012 \\
\hline $\mathrm{Kc2}$ & &
\end{tabular}

$\mathrm{X}$ - dias; EF - eficiência da modelagem; $\mathrm{R}^{2}$ - coeficiente de determinação; EME - erro médio da estimativa 


\section{CONCLUSÕES}

De acordo com os objetivos do trabalho, podese concluir que:

- O tratamento que não teve reposição diária de água apresenta maior precisão, com valores médios de Kc de 0,67; 1,06; 1,12; 0,80, respectivamente, para os estágios I, II, III e IV do feijoeiro semeado em abril;

- Os valores de Kc diferem daqueles propostos por Doorenbos e Kassam (1979).

\section{REFERÊNCIAS BIBLIOGRÁFICAS}

ALLEN, R.G.; PEREIRA, L.S.; RAES, D.; SMITH, M. Crop evapotraspiration: guidelines for computing crop water requirements. Rome: FAO, 1998. 297p.

BASTOS, E.A.; FERREIRA, V.M.; SILVA, C.R.; ANDRADE JÚNIOR, A.S. Evapotranspiração e coeficiente de cultivo do feijão-caupi no Vale do Gurgéia, Piaui. Irriga, Botucatu, v.13, n.2, p.182190, 2008.

BIZARI, D.R.; MATSURA, E.E.; ROQUE, M.W.; SOUZA, A.L. Consumo de água e produção de grãos do feijoeiro irrigado em sistemas plantio direto e convencional. Ciência Rural, Santa Maria, v.39, n.7, p.2073-2079, 2009. Disponível em: $<$ http://www.scielo.br/pdf/cr/v39n7/a18v39n7. pdf $>$. Acesso em: 20 dez. 2015.

\section{COMPANHIA}

NACIONAL

ABASTECIMENTO

-

DE

CONAB.
Acompanhamento da Safra Brasileira de Grãos 2015/2016 - Terceiro Levantamento. Brasília: CONAB, 2015. 147p. Disponível em: < http:// www.conab.gov.br/conteudos.php? $\mathrm{a}=1253 \& \mathrm{t}=>$. Acesso e: 10 jan. 2016.

DOORENBOS, J; KASSAM, A. H. Yield response to water. Roma: FAO, 1979. 193p. (Tecnical note, $33)$.

GUIMARÃES, C.M.; STONE, L.F.; BRUNINI, O. Adaptação do feijoeiro comum (Phaseolus vulgaris
L.) à seca. Revista Brasileira de Engenharia Agrícola e Ambiental, Campina Grande, v.10, n.1, p.70-75, 2006. Disponível em: $<$ http://www.scielo. br/pdf/rbeaa/v10n1/v10n1a11.pdf> . Acesso em: 10 dez. 2015.

LEAL, B.G.; SEDIYAMA, G.C. Modelo matemático para determinação da curva do coeficiente de cultura, Kc. Engenharia na Agricultura, Viçosa, v.12, n.2, p.124-133, 2004.

LIMA, J.R.S.; ANTONINO, A.C.D.; LIRA, C.A.B.O.; SOUZA, E.S.; SILVA, I.F. Balanço de energia e evapotranspiração de feijão caupi sob condições de sequeiro. Revista Ciência Agronômica, Fortaleza, v.42, n.1, p.65-74, 2011. Disponível em: $<$ http://www.ccarevista.ufc.br/ seer/index.php/ccarevista/article/view/1118>. Acesso em: 11 mai. 2016.

MENDONÇA, J.C.; SOUSA, E.F.; BERNARDO, S.; SUGAWARA, M.T.; PEÇANHA, A.L.; GOTTARDO, R.D. Determinação do coeficiente cultural $(\mathrm{Kc})$ do feijoeiro (Phaseolus vulgaris L.), em Campos dos Goytacazes, RJ. Revista Brasileira Engenharia Agrícola e Ambiental, Campina Grande, v.11, n.5, p.471-475, 2007. Disponível em: $<\underline{\text { http://dx.doi.org/10.1590/S1415- }}$ 43662007000500004> . Acesso em: 08 jan. 2016.

MIORINI, T.J.J.; SAAD, J.C.C.; MENEGALE, M.L. Supressão de água em diferentes fases fenológicas do feijoeiro (Phaseolus vulgaris L.). Irriga, Botucatu, v.16, n.4, p.360-368, 2011. Disponível em: <http://dx.doi.org/10.15809/ irriga.2011v16n4p360>. Acesso em: 20 nov. 2015.

OLIVEIRA, P.M. Estimativa da evapotranspiração e do coeficiente de cultura do cafeeiro (Cofeea arabica L). 2003. 90f. Dissertação (Mestrado em Engenharia Agrícola) - Universidade Federal de Lavras, Lavras, 2003.

RAIJ, B. VAN; CANTARELLA, H.; QUAGGIO, J.A.; FURLANI, A.M.C. Recomendações de adubação e calagem para o estado de São Paulo. Campinas: Instituto Agronômico/Fundação IAC, 1997. 285p. 
RIBEIRO, M.S.; SEILA, E.L.; MOURA, D.C.M.; DANTAS, A.A.A. Coeficiente de cultura $(\mathrm{Kc})$ e crescimento vegetativo de acaiá cerrado associados a graus-dia e desenvolvimento. Irriga, Botucatu, v.14, n.2, p.220-232, 2009.

SIMEÃO, M.; OLIVEIRA, A.E.S.; SANTOS, A.R.B.; MOUSINHO, E.P.; RIBEIRO, A.A. Determinação da ETc e Kc para o feijão - fava (Phaseolus lunatus L.) na região de Teresina, Piau. Revista Verde, Pombal, v.8, n.2, p.291-296, 2013. Disponível em: $<$ http://dx.doi.org/10.18378/rvads. v11i2.4173 $>$. Acesso em: 11 mai. 2016.

SILVA, C.M.; ALVES JÚNIOR, J.; SILVA, T.J.A.; FOLEGATTI, M.V.; CAMPECHE, L.F.S.M. Variação sazonal na evapotranspiração de plantas jovens de lima ácida 'tahiti'. Irriga, Botucatu, v.11, n.1, p.26-35, 2006.
SOUZA, A.P.; PEREIRA, J.B.A.; SILVA, L.D.B.; GUERRA, J.G.M.; CARVALHO, D.F. Evapotranspiração, coeficientes de cultivo e eficiência do uso da água da cultura do pimentão em diferentes sistemas de cultivo. Acta Scientiarum. Agronomy, Maringá, v.33, n.1, p.15-22, 2011. Disponível em: $\quad<$ http://dx.doi.org/10.4025/ actasciagron.v33i1.5527>. Acesso em: 03 nov. 2015.

Universidade Estadual Paulista - UNESP - FEIS. Acesso à base diária: Ilha Solteira. Ilha Solteira: UNESP: FE/Departamento de Fitossanidade Engenharia Rural e Solos - DEFERS. Área de Hidráulica e Irrigação - AHI, 2012. Disponível em: $<$ www.agr.feis.unesp.br/irrigacao.php>. Acesso em: 30 mai. 2012. 